\title{
Flexible needle with integrated optical coherence tomography probe for imaging during transbronchial tissue aspiration
}

Jiawen $\mathrm{Li}$

Bryden C. Quirk

Peter B. Noble

Rodney W. Kirk

David D. Sampson

Robert A. McLaughlin 


\title{
Flexible needle with integrated optical coherence tomography probe for imaging during transbronchial tissue aspiration
}

\author{
Jiawen Li, ${ }^{a, b, *}$ Bryden C. Quirk, ${ }^{a, b}$ Peter B. Noble, ${ }^{c, d}$ Rodney W. Kirk, ${ }^{a, b}$ David D. Sampson, ${ }^{\text {e,f }}$ and \\ Robert A. McLaughlin a,b \\ aUniversity of Adelaide, Adelaide Medical School, Australian Research Council Centre of Excellence for Nanoscale Biophotonics, Adelaide, \\ South Australia, Australia \\ bUniversity of Adelaide, Institute for Photonics and Advanced Sensing, Adelaide, South Australia, Australia \\ 'University of Western Australia, School of Human Sciences, Perth, Western Australia, Australia \\ 'University of Western Australia, School of Paediatrics and Child Health, Centre for Neonatal Research and Education, Perth, \\ Western Australia, Australia \\ eUniversity of Western Australia, School of Electrical, Electronic and Computer Engineering, Optical+Biomedical Engineering Laboratory, \\ Perth, Western Australia, Australia \\ fUniversity of Western Australia, Centre for Microscopy, Characterisation and Analysis, Perth, Western Australia, Australia
}

\begin{abstract}
Transbronchial needle aspiration (TBNA) of small lesions or lymph nodes in the lung may result in nondiagnostic tissue samples. We demonstrate the integration of an optical coherence tomography (OCT) probe into a 19-gauge flexible needle for lung tissue aspiration. This probe allows simultaneous visualization and aspiration of the tissue. By eliminating the need for insertion and withdrawal of a separate imaging probe, this integrated design minimizes the risk of dislodging the needle from the lesion prior to aspiration and may facilitate more accurate placement of the needle. Results from in situ imaging in a sheep lung show clear distinction between solid tissue and two typical constituents of nondiagnostic samples (adipose and lung parenchyma). Clinical translation of this OCT-guided aspiration needle holds promise for improving the diagnostic yield of TBNA. ๑ 2017 Society of Photo-Optical Instrumentation Engineers (SPIE) [DOI: 10.1117/1.JBO.22.10.106002]
\end{abstract}

Keywords: optical coherence tomography; fiber-optic imaging; endoscopic imaging; biopsy guidance.

Paper 170411LR received Jun. 23, 2017; accepted for publication Sep. 8, 2017; published online Oct. 11, 2017.

\section{Introduction}

Lung cancer is the most prevalent cancer worldwide and one of the most aggressive, with a 5-year survival rate of $10 \%$ to $15 \%{ }^{1}$ Accurate staging of the disease is critical for appropriate patient management. ${ }^{2,3}$ Staging using imaging alone (such as $\mathrm{x}$-raycomputed tomography or positron emission tomography) is often not adequate, ${ }^{4,5}$ and the gold-standard is histopathological assessment from biopsy. ${ }^{6}$ Transbronchial needle aspiration (TBNA) is a common technique for obtaining cells for histopathological analysis and is associated with low complications. ${ }^{7}$ TBNA is performed by inserting a flexible needle into the working channel of a bronchoscope positioned in the airway lumen, through the airway wall, and into a lesion or lymph node and then aspirating a small volume of tissue. However, accurate positioning of the needle tip relative to the lesion or node is difficult, often resulting in nondiagnostic tissue samples (i.e., samples that do not yield a definitive pathology). ${ }^{8}$ Slight misplacement of the needle tip will position it in nearby uninvolved tissue, such as lung parenchyma ${ }^{9}$ or adipose. ${ }^{10}$ There is clinical evidence that diagnostic yields of both endobronchial ultrasound (EBUS)guided and unguided TBNA are low (as low as 33\% and 14\%, respectively), particularly in pulmonary nodules or lymph nodes smaller than $20 \mathrm{~mm} .^{8,11}$ When performed in medical centers where onsite pathology is not available, this can result in failure to accurately stage the disease, causing under-diagnosis or

*Address all correspondence to: Jiawen Li, E-mail: Jiawen.li01@ adelaide.edu au requiring a repeat procedure. In centers with onsite pathology, nondiagnostic samples may significantly extend the time required for the intervention as the tissue samples are assessed prior to completion of the procedure.

High-resolution optical guidance has the potential to improve the positioning of the needle and increase the diagnostic yield. $^{12-14}$ Optical coherence tomography (OCT) is a medical imaging modality that can provide real-time imaging of tissue microstructure and enable intraoperative guidance with a resolution typically in the range 5 to $20 \mu \mathrm{m} .{ }^{15,16}$ OCT has previously been demonstrated to be capable of differentiating uninvolved and malignant pulmonary tissue. ${ }^{9,17-19}$ Preliminary work has also demonstrated that OCT has the potential for integration into needles for differentiation of tissue types. ${ }^{20-22}$ In particular, a needle probe designed for guiding TBNA ${ }^{21}$ has been demonstrated in a previous study. In that study, a three-stage guiding method was proposed, whereby a catheter-based OCT probe was inserted through the same needle lumen as used for tissue sampling. In the first stage, the needle and the OCT catheter was inserted into the tissue. In the second stage, the TBNA needle was withdrawn, leaving the OCT catheter exposed for 3-D imaging. In the third stage, the TBNA needle was readvanced over the OCT catheter and the catheter was fully withdrawn from the needle lumen, enabling aspiration through the needle. This approach has the advantage of both the OCT probe and aspiration channel being colocated. However, the time delay between imaging and

1083-3668/2017/\$25.00 @ 2017 SPIE 
tissue aspiration combined with respiratory motion presents the risk of dislodging the needle from the lesion prior to aspiration.

Here, we present an alternative design from which imaging and aspiration may be performed simultaneously in a single monolithic flexible needle. Specifically, our proposed needle design includes a second, parallel channel through which tissue can be imaged immediately prior to and during aspiration. By incorporating the imaging probe within the needle, the clinician is able to visualize whether the needle is appropriately lodged within the lymph node or lesion to a level of accuracy not possible with solely ultrasound guided or unguided TBNA. Although dual-channel needle designs have been proposed for other applications, ${ }^{23,24}$ these designs are too rigid to be used within a flexible bronchoscope and, hence, are not suitable for TBNA. We illustrate the feasibility of this approach with an in situ experiment in a sheep lung and show clear distinction between solid tissue, adipose, and lung parenchyma. The capacity to identify adipose and lung parenchyma, which are typical constituents of nondiagnostic samples, ${ }^{9,10,25}$ provides an opportunity for OCT needles to avoid sampling these normal tissues and improve the diagnostic yield during TBNA. To the best of our knowledge, this is the first demonstration of a flexible endoscopic needle with integrated OCT imaging capable of simultaneous imaging and tissue aspiration.

\section{Materials and Methods}

The flexible integrated OCT and aspiration needle was fabricated by modifying a standard Olympus flexible endoscopic needle (19-gauge aspiration needle, NA-220H-8019, Olympus, Japan), shown in Fig. 1(a). Two parallel channels, for imaging and aspiration, were created within the lumen of the needle, as shown schematically in Figs. 1(b) and 1(c). The inner diameter of the aspiration channel is comparable to that of the 22-gauge TBNA needle commonly used in clinical practice. A $650-\mu \mathrm{m}$ imaging window was machined into the needle's stainless-steel wall using electrical discharge machining. To avoid compromising the structural integrity of the needle tip, the hole was offset from the end of the sharpened needle tip by $1.35 \mathrm{~mm}$. This offset is considerably smaller than the 5- to $20-\mathrm{mm}$ diameter lesions and lymph nodes potentially targeted for aspiration.

The imaging channel comprises a miniaturized all-fiber OCT probe based on the design described previously. ${ }^{26}$ A 2-m length of single-mode fiber was distally terminated with a $420-\mu \mathrm{m}$ length of no-core fiber (POFC, Taiwan), allowing the light beam to expand, and then spliced to a 200- $\mu \mathrm{m}$ section of GRIN fiber (DrakaElite, The Netherlands) to focus the beam. This design results in an expected OCT working distance of $0.45 \mathrm{~mm}$ and a focal spot size of $14 \mu \mathrm{m}$ (in air). The GRIN fiber section was terminated with an angle-polished section of no-core fiber to create total internal reflection (TIR) at its interface to air. The beam was directed at an 8 deg side-viewing angle, illustrated schematically in Fig. 1(b). The optical assembly was encased within a medical-grade polyimide tube [inner diameter (ID): $270 \mu \mathrm{m}$; outer diameter (OD): $295 \mu \mathrm{m}$, MicroLumen] and rigidly affixed within the needle using optical adhesive (NOA81, Norland Products). The adhesive was cured with UV light through both the needle tip and imaging window. We found this approach to be mechanically robust, although we note that combined heat-and-UV-cured adhesives may provide a more robust prototype. The polyimide tubing was necessary to keep the angle-polished no-core fiber clear of optical adhesive to maintain TIR and to improve robustness of the fiber probe during insertion and positioning inside the needle.

The parallel aspiration channel was fabricated within the needle using a second length of medical-grade polyimide tube (ID: $410 \mu \mathrm{m}$; OD: $435 \mu \mathrm{m}$, MicroLumen), chosen for its high tensile strength. The imaging probe and aspiration channel were positioned to be parallel within the needle lumen. To avoid damaging the delicate focusing optics during insertion, we inserted the optical fiber assembly from the distal end of the needle and then

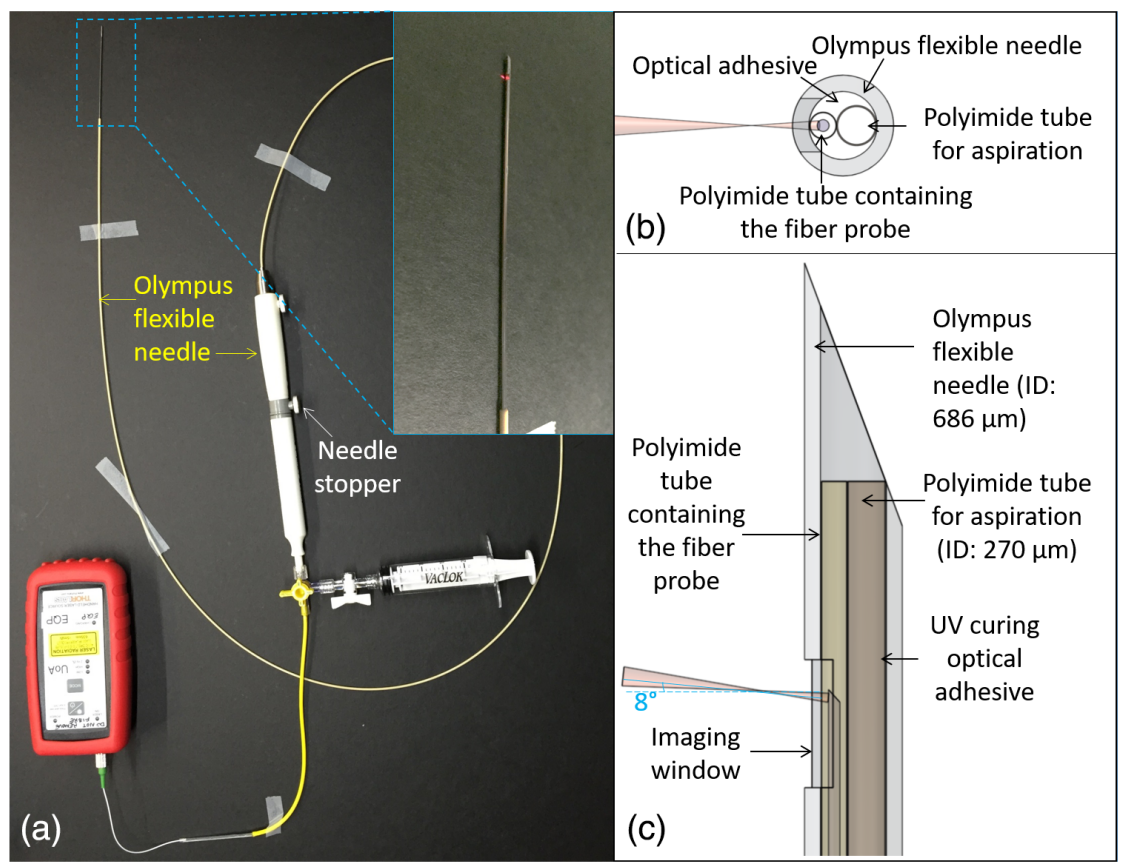

Fig. 1 (a) Photo of the flexible integrated OCT and aspiration biopsy needle with test light source attached. Inset: a close-up view of the needle tip. Schematic of the needle tip and beam: (b) crosssectional view and (c) front view. 
retracted it into position. A three-way stopcock was attached at the proximal end of the needle, as shown in Fig. 1(a), to guide the proximal end of the fiber to be interfaced to the OCT scanner and allow a syringe to be connected to create negative pressure and facilitate tissue aspiration.

As the needle is embedded interstitially within the tissue during TBNA procedures, movement is naturally restricted to one dimension, and manual translation along the axis of needle movement acquires a longitudinal B-scan. The resulting 2-D image shows the tissue microarchitecture immediately adjacent to the needle, allowing differentiation between tissue types. Note that, by avoiding the inclusion of additional scanning mechanisms, we provide for ready miniaturization, as well as robustness of the probe, and reduce issues caused by tissue drag. ${ }^{27}$

We used the light source, data acquisition card, and workstation of a commercially available spectral-domain OCT system (Telesto II, Thorlabs), with a central wavelength of $1300 \mathrm{~nm}$ and a manufacturer-specified axial resolution of $5.5 \mu \mathrm{m}$ (in air). This system was interfaced to a custom-built interferometer that was developed in-house, which included our flexible-integrated OCT and aspiration needle as the sample arm. A-scans were acquired at $48 \mathrm{kHz}$.

Animal tissue experiments were approved under tissue sharing protocols of The University of Western Australia Animal Ethics Committee. In situ imaging experiments were performed in a sheep airway within 2 hours postmortem. Since the sheep trachea is longer than a human trachea, a tracheotomy was performed, so a standard clinical bronchoscope could be inserted deeper into the lung. The bronchoscope was navigated to the regions of interest and positioned against the airway wall. The flexible needle was inserted into the working channel of the bronchoscope and directed to pierce the airway wall. Insertions were performed manually. OCT images were recorded, and provided continuous, real-time feedback on tissue structures adjacent to the needle. After assessing the real-time OCT images to confirm needle positioning, aspiration was conducted using the syringe connected to the side-port of the three-way stopcock, as shown in Fig. 1(a). The location of each insertion was recorded using airway bifurcations as landmarks. At the completion of imaging, an excisional biopsy was performed at each location of needle insertion to allow histological processing of a representative section of tissue. We note that, in clinical practice, the tissue sample will be obtained only through the built-in aspiration channel. However, as aspiration degrades the microarchitecture of the tissue, we chose to match samples against excisional biopsy for this proof of concept study.

\section{Results and Discussion}

OCT images acquired with the flexible needle are shown in Fig. 2, paired with representative histology sections from the excisional biopsy. In these images, the needle is located at the top of the image, translating left to right, with axial distance increasing from top to bottom in each image. Adipose tissue [Fig. 2(a)] shows multiple interfaces from the cellular membranes encompassing lipid-rich regions, in agreement with earlier work. ${ }^{28}$ As lung parenchyma [Fig. 2(b)] comprises predominantly air-filled alveoli, it presents as a highly backscattering tissue-air interface and reveals little additional structure. ${ }^{23}$ In addition, as the needle passes through air-filled parenchyma regions, the OCT shows only the residual tissue on the imaging window that is stationary, resulting in characteristically long speckles in the lateral direction. These results correlate well

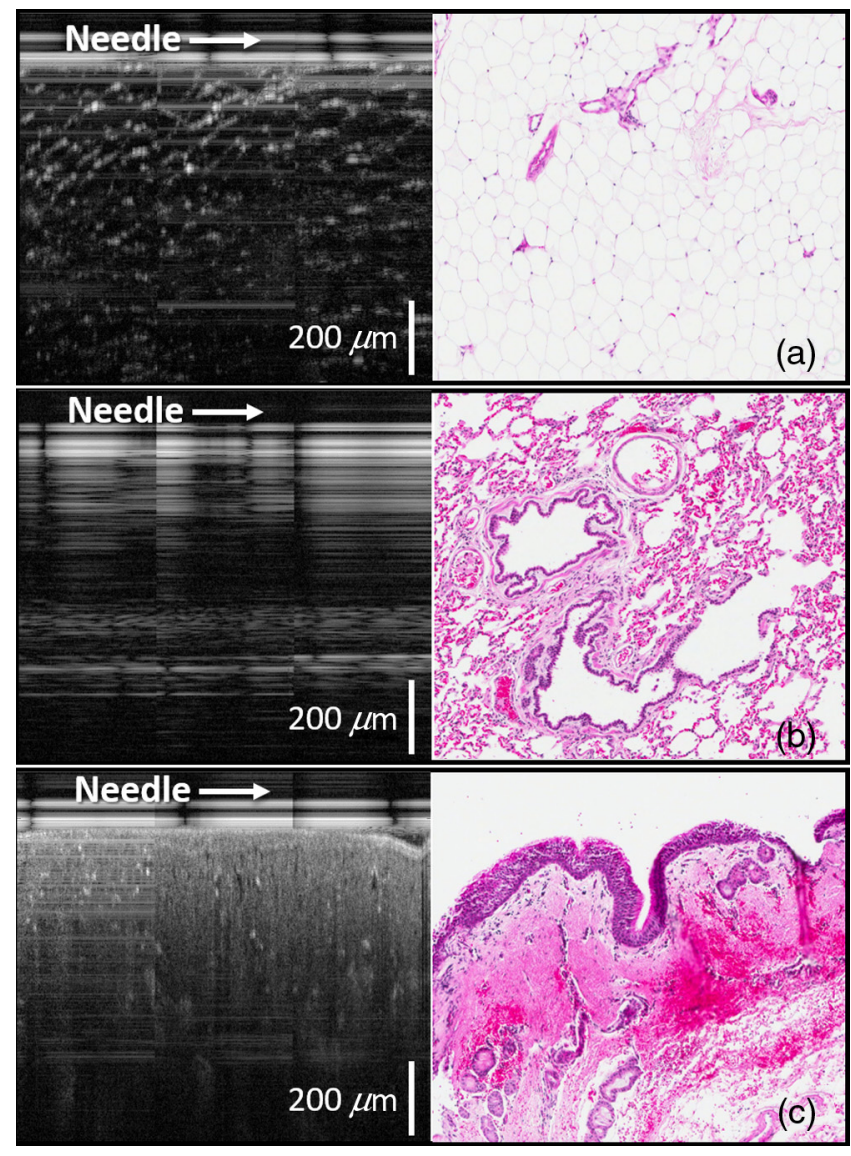

Fig. 2 OCT longitudinal B-scans of (a) adipose, (b) alveoli, and (c) solid tissue. Right panels show representative H\&E histology. The axial scale bar shows physical distance. The lateral scale varies with manual scan speed.

with previous findings. ${ }^{29}$ Solid tissue is characterized by homogeneous areas of backscatter. Figure 2(c) presents an example of the image from solid tissue punctuated by small voids typical of tubular structures such as small vessels. The small number of spurious horizontal lines visible in the images is an artifact of the background subtraction algorithm. The axial scale bar shows physical distance, assuming an average value for the refractive index of lung tissue of 1.38 (as measured at $633 \mathrm{~nm}$ in Ref. 30). Detailed interpretation of the structural features present is complicated by both OCT imaging artifacts and effects of the needle insertion and will require larger studies to define appropriate criteria, such as those presented by Hariri et al. ${ }^{9}$

Longitudinal B-scans (Fig. 3) were constructed as the needle was manually translated interstitially. As the B-scan imaging was not synchronized with the needle insertion, distortion can be observed in the form of areas of elongated speckle, comparable to the artifacts seen in nonuniform rotation distortion present in rotational OCT scanning. These discontinuities could possibly be reduced, if necessary, using a realtime distortion correction technique based on the statistics of speckle between A-lines. ${ }^{31}$ Further, in interpreting the videos, it is important to note that the lateral extent of the B-scan is only approximately known and the lateral scale varies with the velocity of needle insertion. Figure 3(a) shows images acquired as the needle was inserted into regions of solid tissue. The video extends over an axial depth of $0.95 \mathrm{~mm}$. Several structures are present in the video, including a cross-sectional 

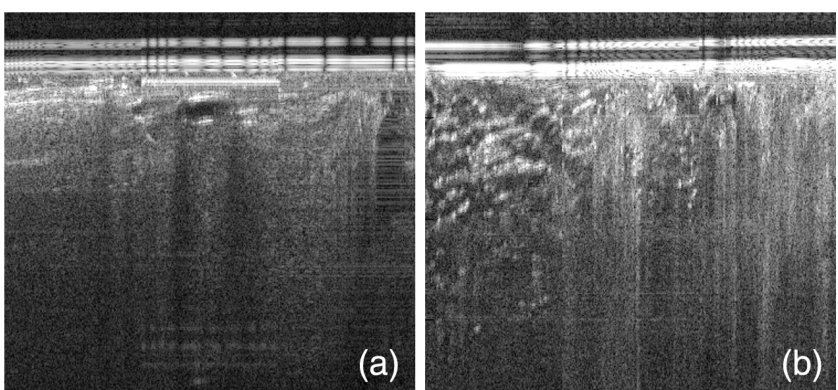

Fig. 3 Longitudinal B-scans acquired as the needle was inserted into regions of (a) solid tissue (Video 1, MOV, 2 MB [URL: http://dx doi.org/10.1117/1.JBO.JBO.22.10.106002.1]) and (b) adipose tissue (Video 2, MOV, 2 MB [URL: http://dx.doi.org/10.1117/1. JBO.22.10.106002.2]).

view of a lumen visible at $10 \mathrm{~s}$, likely to be an air-filled bronchiole. Figure 3(b) shows a B-scan constructed during insertion into a region of adipose. The image is characterized by the honeycomb-like structure from the adipose cell walls, and low backscatter from the intracellular lipid, providing a marked distinction from areas of solid tissue that may be diagnostic. This is replaced by regions of more homogeneous backscatter as the needle passes through the adipose and into solid tissue.

Unlike our earlier works, ${ }^{27,28}$ no saline or other refractiveindex matching fluid was used in this experiment, so as to closely mimic the air-filled conditions in vivo. We note that the goal of our previous studies was to form detailed images of tissue morphology, whereas the clinical goal here is to identify when the needle is erroneously positioned. The lack of refractive-index matching fluid was found to emphasize the distinction between air-filled lung parenchyma [Fig. 2(b)] and other tissue types, such as adipose [Fig. 2(a)] or solid [Fig. 2(c)] tissue.

The integrated design presented in this paper allows OCT guidance for tissue identification during a needle aspiration procedure. While the integrated OCT and aspiration needle design results in a larger bore device than a stand-alone needle, it has the potential to reduce the number of nondiagnostic aspiration samples. Preclinical in vivo studies, imaging of different lesion types, and corresponding statistical analyses are now required to evaluate whether OCT-guided imaging can improve the diagnostic yield of TBNA.

TBNA procedures may use either a 19-, 21-, or 22-gauge flexible needle, ${ }^{32}$ depending on the amount of tissue required and aspiration location. The current study utilized a 19-gauge needle, but the design is applicable to smaller needles as further miniaturization of the imaging channel can be achieved. ${ }^{26}$ The range of potential needle sizes opens the possibility of generalizing this design to other organs. While we have focused on the application of staging of lung disease, we believe that this integrated imaging-aspiration design has applications in tissue aspiration in other areas, such as the gastrointestinal tract, the pancreas, and bile ducts.

\section{Conclusion}

We have presented a 19-gauge flexible, integrated OCT and aspiration needle that provides real-time OCT images to identify the tissue type surrounding the needle tip, immediately prior to and during aspiration, without requiring TBNA needle retraction and replacement or imaging probe withdrawal. Preliminary results from an in situ sheep lung experiment show clear distinction between solid tissue and typical constituents of non-diagnostic samples (such as adipose and lung parenchyma). We anticipate that the clinical translation of this needle design could increase the diagnostic yield of TBNA.

\section{Disclosures}

The authors have no relevant financial interests in this article and no potential conflicts of interest to disclose.

\section{Acknowledgments}

This work was supported by the Australian Research Council (CE140100003 and DP150104660), the National Health \& Medical Research Council (Australia) Career Development Fellowship \#1045824 and Development Grant \#1076050, and a Premier's Research and Industry Fund grant provided by the South Australian Government Department of State Development.

\section{References}

1. B. W. Stewart and C. P. Wild, "World cancer report 2014," International Agency for Research on Cancer, Lyon (2014).

2. F. C. Detterbeck et al., "Executive summary: diagnosis and management of lung cancer, 3rd ed: American College of Chest Physicians evidencebased clinical practice guidelines," Chest 143(5 Suppl.), 7S-37S (2013).

3. G. R. Simon and A. Turrisi, "Management of small cell lung cancer: ACCP evidence-based clinical practice guidelines (2nd edition)," Chest 132(3 Suppl.), 324S-339S (2007).

4. Y. T. Sim and F. W. Poon, "Imaging of solitary pulmonary nodule: a clinical review," Quant. Imaging Med. Surg. 3(6), 316-326 (2013).

5. H. MacMahon et al., "Guidelines for management of small pulmonary nodules detected on CT scans: a statement from the Fleischner Society," Radiology 237(2), 395-400 (2005).

6. T. I. Powell et al., "Peripheral lung nodules: fluoroscopically guided video-assisted thoracoscopic resection after computed tomographyguided localization using platinum microcoils," Ann. Surg. 240(3), 481-489 (2004)

7. F. Asano et al., "Complications associated with endobronchial ultrasound-guided transbronchial needle aspiration: a nationwide survey by the Japan Society for Respiratory Endoscopy," Respir. Res. 14(1), 1-8 (2013).

8. L. P. Hariri et al., "In vivo optical coherence tomography: the role of the pathologist," Arch. Pathol. Lab. Med. 136(12), 1492-1501 (2012).

9. L. P. Hariri et al., "Toward the guidance of transbronchial biopsy: identifying pulmonary nodules with optical coherence tomography," Chest 144(4), 1261-1268 (2013).

10. W. E. Khalbuss, "Normal and nonneoplastic components," in Endobronchial Ultrasound-Guided Transbronchial Needle Aspiration (EBUS-TBNA): A Practical Approach, S. E. Monaco, W. E. Khalbuss, and L. Pantanowitz, Eds., p. 62, Karger Medical and Scientific Publishers, Basel (2014).

11. M. P. Rivera and A. C. Mehta, "Initial diagnosis of lung cancer: ACCP evidence-based clinical practice guidelines (2nd edition)," Chest 132(3 Suppl.), 131S-148S (2007).

12. W.-C. Kuo et al., "Real-time three-dimensional optical coherence tomography image-guided core-needle biopsy system," Biomed. Opt. Express 3(6), 1149-1161 (2012).

13. N. V. Iftimia et al., "Spectral-domain low coherence interferometry/ optical coherence tomography system for fine needle breast biopsy guidance," Rev. Sci. Instrum. 80(2), 024302 (2009).

14. N. V. Iftimia et al., "A portable, low coherence interferometry based instrument for fine needle aspiration biopsy guidance," Rev. Sci. Instrum. 76(6), 064301 (2005).

15. D. Huang et al., "Optical coherence tomography," Science 254(5035), 1178-1181 (1991)

16. P. Hahn et al., "The use of optical coherence tomography in intraoperative ophthalmic imaging," Ophthalmic Surg. Lasers Imaging Retina 42(0), S85-S94 (2011).

17. L. P. Hariri et al., "Seeing beyond the bronchoscope to increase the diagnostic yield of bronchoscopic biopsy," Am. J. Respir. Crit. Care Med. 187(2), 125-129 (2013). 
18. R. G. Michel et al., "Optical coherence tomography as an adjunct to flexible bronchoscopy in the diagnosis of lung cancer: a pilot study," Chest 138(4), 984-988 (2010).

19. S. Lam et al., "In vivo optical coherence tomography imaging of preinvasive bronchial lesions," Clin. Cancer Res. 14(7), 2006-2011 (2008).

20. Y. Wu et al., "Robust high-resolution fine OCT needle for side-viewing interstitial tissue imaging," IEEE J. Sel. Top. Quantum Electron. 16(4), $863-869$ (2010)

21. K. M. Tan et al., "Flexible transbronchial optical frequency domain imaging smart needle for biopsy guidance," Biomed. Opt. Express 3(8), 1947-1954 (2012).

22. R. A. McLaughlin et al., "Imaging of breast cancer with optical coherence tomography needle probes: feasibility and initial results," IEEE $J$. Sel. Top. Quantum Electron. 18(3), 1184-1191 (2012).

23. B. C. Quirk et al., "Optofluidic needle probe integrating targeted delivery of fluid with optical coherence tomography imaging," Opt. Lett. 39(10), 2888-2891 (2014).

24. M. S. Jafri, R. Tang, and C.-M. Tang, "Optical coherence tomography guided neurosurgical procedures in small rodents," J. Neurosci. Methods 176(2), 85-95 (2009).

25. S. E. Monaco, W. E. Khalbuss, and L. Pantanowitz, Endobronchial Ultrasound-Guided Transbronchial Needle Aspiration (EBUS-TBNA):
A Practical Approach, Karger Medical and Scientific Publishers, Basel (2014).

26. D. Lorenser et al., "Ultrathin side-viewing needle probe for optical coherence tomography," Opt. Lett. 36(19), 3894-3896 (2011).

27. R. A. McLaughlin et al., "Static and dynamic imaging of alveoli using optical coherence tomography needle probes," J. Appl. Physiol. 113(6), 967-974 (2012).

28. B. C. Quirk et al., "In situ imaging of lung alveoli with an optical coherence tomography needle probe," J. Biomed. Opt. 16(3), 036009 (2011).

29. R. A. McLaughlin, P. B. Noble, and D. D. Sampson, "Optical coherence tomography in respiratory science and medicine: from airways to alveoli," Physiology 29(5), 369-380 (2014).

30. F. P. Bolin et al., "Refractive index of some mammalian tissues using a fiber optic cladding method," Appl. Opt. 28(12), 2297-2303 (1989).

31. N. Uribe-Patarroyo and B. E. Bouma, "Rotational distortion correction in endoscopic optical coherence tomography based on speckle decorrelation," Opt. Lett. 40(23), 5518-5521 (2015).

32. C. Chen and J.-A. Huang, "How to increase the yield of transbronchial needle aspiration (TBNA)?" J. Thorac. Dis. 7(Suppl. 4), S238-S245 (2015).

Biographies for the authors are not available. 